\title{
Transferencia de Impulsos y Ganancia de Tensión en Transformadores Monofásicos de Baja Potencia
}

\author{
Esteban Velilla, John E. Muñoz, Edwar A. Ramírez, Jaime A. Valencia \\ Univ. de Antioquia, Fac. de Ingeniería, Depto Ing. Eléctrica, Grupo de Manejo Eficiente de la \\ Energía - Gimel, Calle 67 No. 53-108, Oficina 19-443, Medellín-Colombia \\ (e-mail: evelilla@udea.edu.co)
}

Recibido May. 24, 2011; Aceptado Jul. 19, 2011; Versión Final recibida Ago. 25, 2011

\begin{abstract}
Resumen
Se evaluó la transferencia de impulsos de tensión $1.2 / 50 \mu$ s y $3 / 23 \mu$ s del primario al secundario, en tres transformadores monofásicos utilizados en aplicaciones electrónicas, uno de $18 \mathrm{~W}$, otro de $85 \mathrm{~W}$ y un autotransformador de $50 \mathrm{~W}$, considerándolos en vacío y con carga resistiva en el secundario. El autotransformador fue el que presentó mayor amortiguamiento y menor valor máximo en el impulso transferido. La máxima transferencia de tensión ocurrió en vacío y se analizaron las respuestas en frecuencia de la ganancia de tensión de los transformadores en vacío entre $10 \mathrm{~Hz}$ y $1 \mathrm{MHz}$, ajustándolas a funciones de transferencia para modelar la transferencia de impulsos de tensión. La confrontación de los resultados experimentales y teóricos tanto en el dominio del tiempo como en el dominio de la frecuencia, mostraron información valiosa sobre la transferencia de los impulsos de tensión en los transformadores monofásicos.
\end{abstract}

Palabras clave: transformador monofásico, impulsos de tensión, ganancia de tensión, respuesta en frecuencia, función de transferencia.

\section{Impulse Transfer and Voltage Gain in Single Phase Low Power Transformers}

\begin{abstract}
The voltage impulse transferences of $1.2 / 50 \mu$ s and $3 / 23 \mu$ s from the primary to the secondary winding was tested in three single phase transformers used in electronic applications; two transformers of $85 \mathrm{~W}$ and $18 \mathrm{~W}$, and one autotransformer of $50 \mathrm{~W}$ were used under no load and under resistive load conditions. The autotransformer showed higher damping and lower peak value in the transferred voltage impulse than the other transformers. The maximum voltage value in the secondary winding was obtained at no load condition and the frequency response of the voltage gain of the transformers between $10 \mathrm{~Hz}$ and $1 \mathrm{MHz}$ were analyzed and fitted to transfer functions to model the voltage impulse transferences. The comparison of experimental results with those obtained from transfer functions in the frequency and time domain provides valuable information about the transferred voltage impulses in single phase transformers.
\end{abstract}

Keywords: single phase transformer, voltage impulse, voltage gain, frequency response, transfer function 


\section{INTRODUCCIÓN}

Las descargas eléctricas atmosféricas (DEA) están entre las principales causas de disturbios en los sistemas eléctricos, pudiendo impactarlos directa o indirectamente. En uno u otro caso, la consecuencia más importante es la aparición de impulsos de tensión en la red caracterizados por el tiempo de frente y de cola del orden de los $\mu \mathrm{s}$, estos impulsos pueden ser transferidos a los diferentes niveles de tensión del sistema eléctrico a través de los transformadores. En el caso de la distribución de energía, los impulsos generados en el lado de media tensión pueden verse reflejados en el lado de baja tensión donde están conectados los usuarios finales, ocasionando en algunos casos, lesiones a personas, daños a instalaciones o equipos, o interrupciones no programadas del suministro de la energía eléctrica.

Dentro de los usuarios finales se encuentra el sector residencial, el cual típicamente utiliza equipos electrónicos que poseen transformadores monofásicos para reducir o aumentar la tensión de la red eléctrica a la tensión de operación de los dispositivos electrónicos. Tanto el aislamiento del transformador como los dispositivos conectados a éstos, pueden verse comprometidos cuando una perturbación es transferida a través del transformador de distribución a la red doméstica, siendo el daño de equipos electrónicos uno de los reclamos más frecuentes de los usuarios a las empresas de distribución, lo cual se ve agudizado en Colombia, en donde la actividad eléctrica atmosférica (rayos) es muy frecuente (NTC 4552-1, 2008).

En la literatura técnica se encuentran varios trabajos que tratan de evaluar y modelar el comportamiento de los transformadores en cuanto a la transferencia de impulsos de tensión debidos a DEA, siendo el tema generalmente abordado desde el punto de vista de transformadores trifásicos (Okabe et al, 2011; De Conti y Visacro, 2005; Obase, 2004; Noda et al, 2004; Das, 2003), evaluando la transferencia de tensión ante la onda de tensión normalizada $1.2 / 50 \mu \mathrm{s}$ u otras ondas que tratan de representar el comportamiento de tensiones inducidas por DEA (Kanashiro et al, 2004), representando convencionalmente la impedancia del transformador a través de modelos circuitales. Por otra parte, la técnica del análisis de respuesta en frecuencia se ha utilizado en el diagnóstico de fallas en transformadores a partir de la comparación de varios registros de respuestas en frecuencia (Secue, 2008). Este trabajo, abordó el tema de la transferencia de tensión, evaluando el comportamiento de transformadores monofásicos de baja potencia utilizados en aplicaciones electrónicas ante excitaciones tipo impulsos de tensión, considerando tanto el vacío como carga resistiva en el lado de baja. Estos resultados mostraron que la máxima transferencia de tensión se presentó cuando los transformadores están en vacío, y con el objetivo de evaluar la mayor tensión transferida al secundario que podría comprometer el aislamiento, y por ende la vida útil de los transformadores, se realizó la respuesta en frecuencia (RF) de la ganancia en tensión vista entre el secundario con respecto al primario en vacío, entre 0 $\mathrm{Hz}$ y $1 \mathrm{MHz}$. Las RF fueron ajustadas a funciones de transferencia (FT) y evaluadas ante impulsos de tensión. La confrontación de los resultados obtenidos en el dominio del tiempo con los impulsos de tensión aplicados, evidenció la importancia de la RF; de la carga en el secundario y de la forma de onda del impulso aplicado, en el impulso transferido al secundario.

\section{IMPULSOS TRANSFERIDOS CONSIDERANDO CARGA RESISTIVA}

Con la finalidad de evaluar la transferencia de impulsos de tensión del lado de alta al lado de baja tensión, y el efecto de la carga resistiva en el secundario de 3 transformadores monofásicos utilizados en aplicaciones electrónicas: uno de $85 \mathrm{~W}$, diseñado para soportar $15 \mathrm{~A}$ en el secundario con relación nominal de tensión de 120/17 V; un autotransformador de 50 W con relación nominal de tensión de 220/120 V, y un transformador de $18 \mathrm{~W}$ con relación nominal de tensión de 120/18 V (Fig. 1), se desarrollo un programa computacional en Matlab (Velilla, 2011), que permite controlar y sincronizar el generador de señales AFG3021B y el osciloscopio digital TDS2022B, ambos marca Tektronix (Fig. 2a). A través del programa se establecen los parámetros de la señal deseada que será aplicada al primario de los transformadores y observada en el secundario, ambas señales son capturadas por el programa computacional a través del osciloscopio. En la Fig. $2 \mathrm{~b}$ se presentan los 2 impulsos de tensión de amplitud $2 \mathrm{~V}$ pico-pico

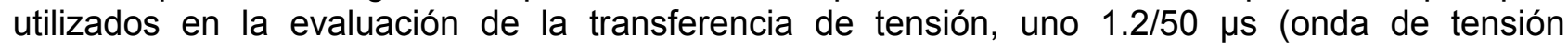


normalizada para DEA), caracterizada por el tiempo de frente rápido, y otro 3/23 $\mu$ s que posee un tiempo de frente mayor y tiempo de cola menor (más rápido) al impulso de tensión normalizado. Estas ondas pretenden representar las formas de ondas de tensión generadas por DEA.

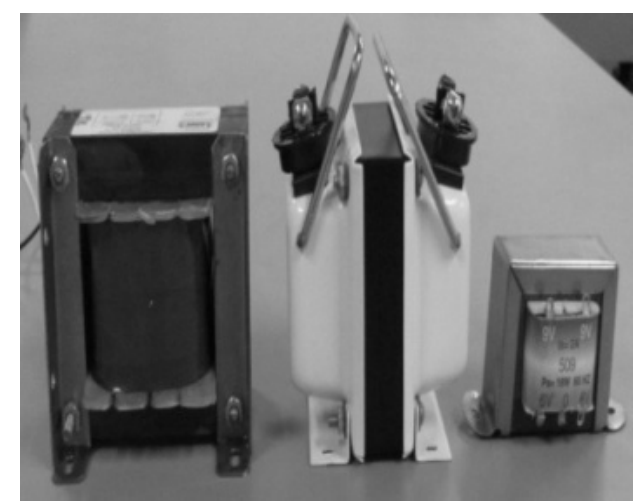

Fig. 1: Transformador de 85,50 y $18 \mathrm{~W}$, respectivamente

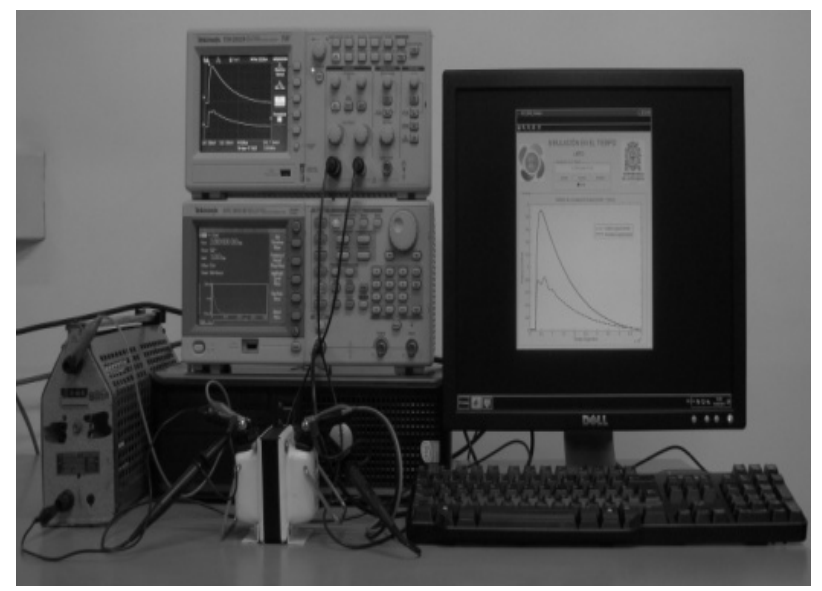

a) Esquema experimental

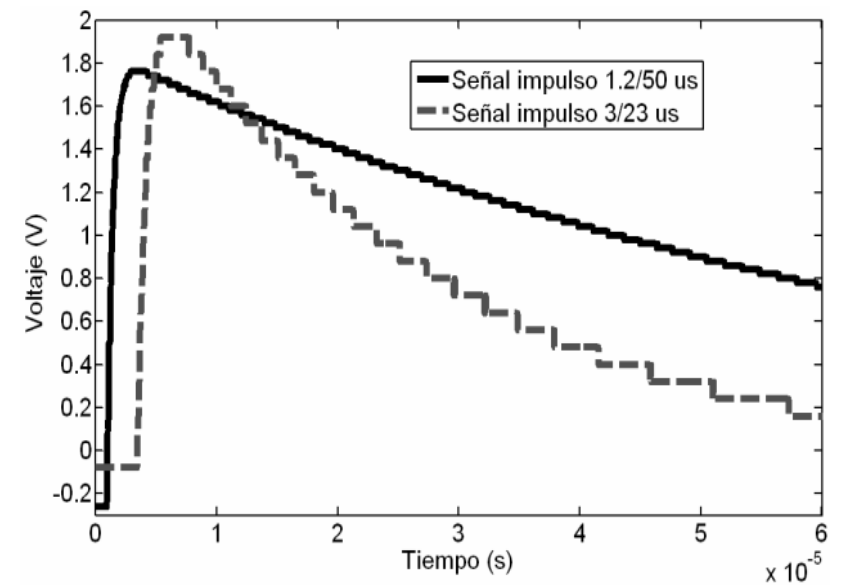

b) Ondas de tensión tipo impulso utilizadas

Fig. 2: Esquema experimental

En las Fig. 3, 4 y 5, se presentan los impulsos de tensión transferidos en el secundario de los 3 transformadores (85, 50 y $18 \mathrm{~W}$ respectivamente), al aplicar los impulsos de tensión mostrados en la Fig. 2b al lado primario de éstos (Fig. 2a), considerando 4 condiciones diferentes: vacío y 3 diferentes cargas resistivas en el secundario de 16, 10 y $4 \Omega$. En estas figuras se observa que los mayores valores de voltaje vistos en el secundario, se dan cuando los transformadores están en vacío, y en la medida en que el valor de la resistencia de la carga en el secundario disminuye, la magnitud del impulso transferido disminuye, comportamiento similar al presentado en (Obase, 2004) para transformadores trifásicos. Adicionalmente se observa que los impulsos transferidos en el autotransformador, son los más lentos y de mayor amortiguamiento para ambas señales de prueba. Por otro lado, a pesar de que las relaciones nominales de tensión de los transformadores de 85 y $18 \mathrm{~W}$ son aproximadamente iguales (120/17 y 120/18, respectivamente), y de que los impulsos transferidos en vacío presentan comportamientos similares en cuanto a las magnitudes y formas de onda, los impulsos transferidos en el transformador de $18 \mathrm{~W}$ son más afectados por la resistencia de carga en el secundario, indicando esto, que el transformador de $85 \mathrm{~W}$ es más robusto, lo cual es evidenciado físicamente en el tamaño (Fig. 1), siendo éste el reflejo de sus características constructivas (el transformador de $85 \mathrm{~W}$ soporta hasta $15 \mathrm{~A}$ en las bobinas del secundario, mientras que el transformador de $18 \mathrm{~W}$, soporta hasta $2 \mathrm{~A}$ ). 


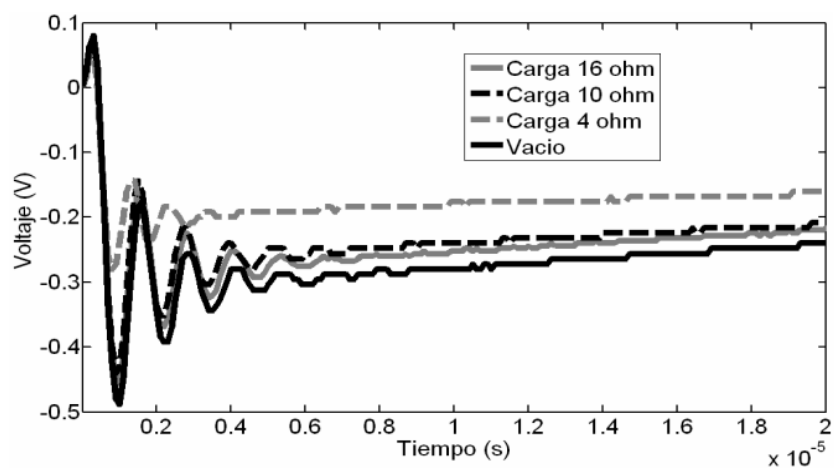

a) Impulso $1.2 / 50 \mu \mathrm{s}$

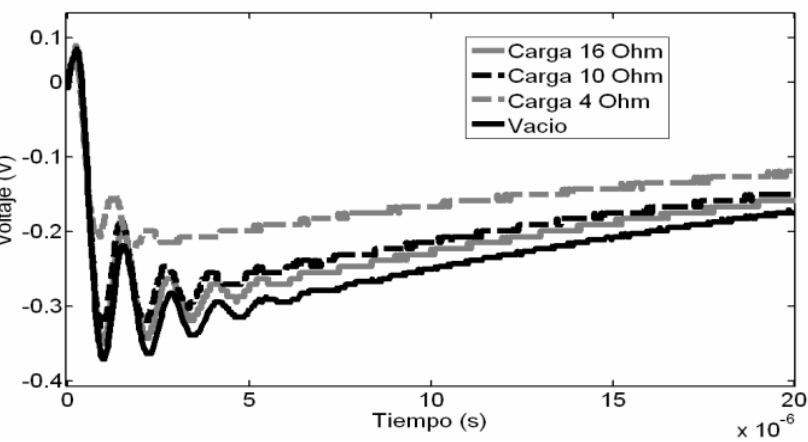

b) Impulso $3 / 23 \mu \mathrm{s}$

Fig. 3: Impulso transferido en el transformador de $85 \mathrm{~W}$

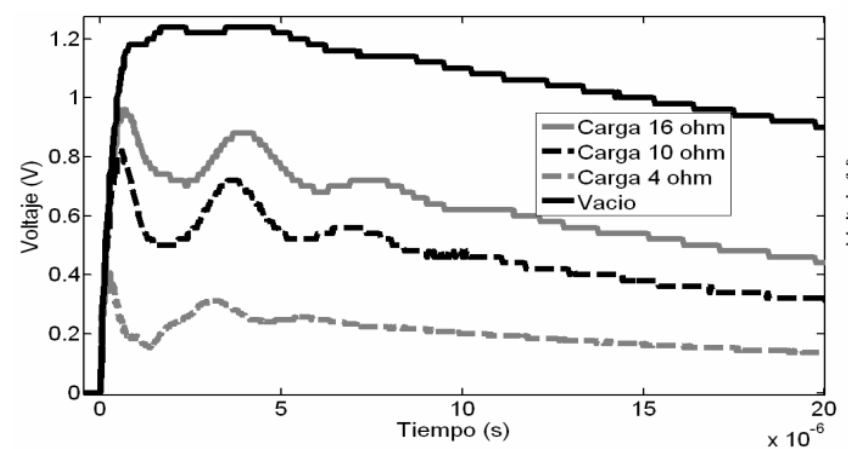

a) Impulso $1.2 / 50 \mu \mathrm{s}$

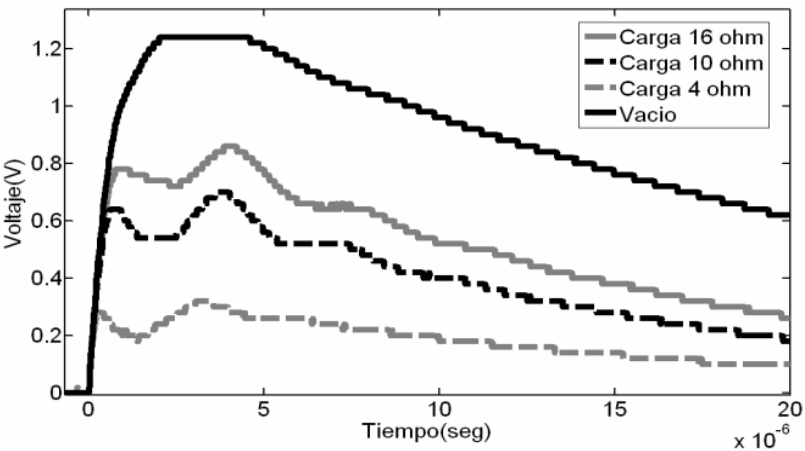

b) Impulso $3 / 23 \mu \mathrm{s}$

Fig. 4: Impulsos transferidos en el autotransformador de $50 \mathrm{~W}$

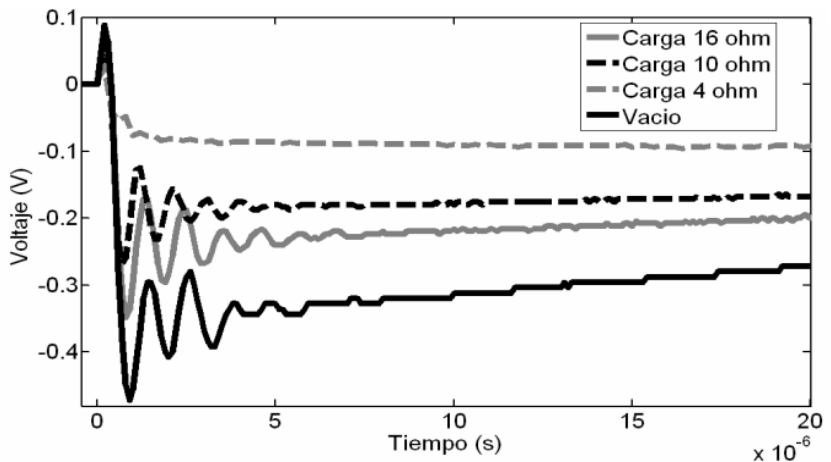

a) Impulso $1.2 / 50 \mu \mathrm{s}$

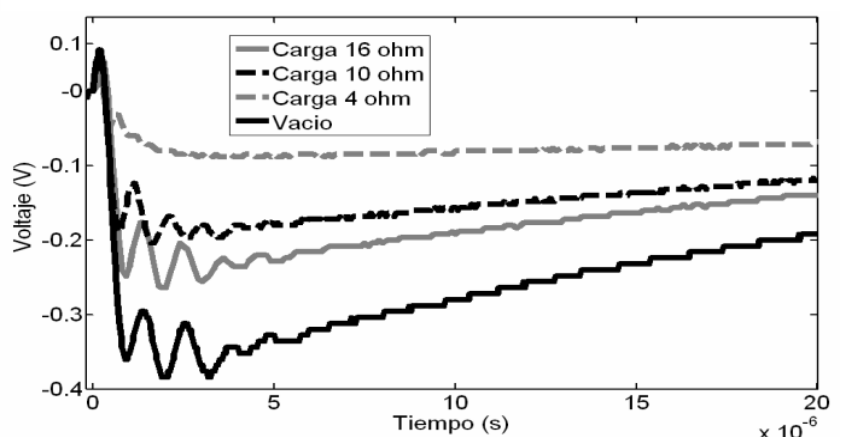

b) Impulso $3 / 23 \mu \mathrm{s}$

Fig. 5: Impulsos transferidos en el transformador de $18 \mathrm{~W}$

En la Tabla 1 se presenta una comparación de los máximos voltajes picos obtenidos en las Fig. 3 , 4 y 5 para las 2 ondas de prueba, observándose que los mayores voltajes pico de los impulsos transferidos ocurren cuando la señal de excitación posee el tiempo de frente más rápido (señal 1.2 $\mu \mathrm{s})$. Adicionalmente, al normalizar los máximos valores pico de tensión con respecto a la relación nominal de transformación (RNT $=V_{\text {out }} / N_{\text {in }}$ ) de cada transformador, si la RNT aumenta, la relación pico máximo de tensión con respecto a la RNT disminuye, razón por la cual el transformador de 85 W al tener la menor RNT (0.14), es el que presenta la mayor relación para ambas señales (3.485 y 2.6, respectivamente), mientras que el autotransformador de $50 \mathrm{~W}$ al poseer la mayor RNT (0.54), presenta la menor relación (2.2963), valor que coincide para ambas señales de prueba. 
Tabla 1: Resultados en el dominio del tiempo

\begin{tabular}{|l|l|l|l|}
\hline Transformadores & $85 \mathrm{~W}$ & $50 \mathrm{~W}$ & $18 \mathrm{~W}$ \\
\hline Relación nominal de Transformación $\left(\mathrm{RNT}=\mathrm{V}_{\text {out }} / \mathrm{V}_{\text {in }}\right)$ & 0.140 & 0.540 & 0.150 \\
\hline Pico máximo en el secundario, onda $1.2 / 50 \mu \mathrm{s}$ & 0.488 & 1.240 & 0.472 \\
\hline Pico máximo en el secundario, onda $3 / 23 \mu \mathrm{s}$ & 0.364 & 1.240 & 0.384 \\
\hline Relación pico máximo / RNT, señal $1.2 / 50 \mu \mathrm{s}$ & 3.485 & 2.296 & 3.146 \\
\hline Relación pico máximo / RNT, señal $3 / 23 \mu \mathrm{s}$ & 2.600 & 2.296 & 2.560 \\
\hline
\end{tabular}

\section{RESPUESTA EN FRECUENCIA DE LA GANANCIA EN TENSIÓN}

Una vez observado el comportamiento en el tiempo de la transferencia de impulsos de tensión en los 3 transformadores, en el cual la máxima transferencia del impulso de tensión, ocurrió en la condición de vacío, y considerando que ésta sería la peor condición a la que estaría sometido el aislamiento de los transformadores y por ende, la vida útil de éstos, se determinó la respuesta en frecuencia de la ganancia de tensión del voltaje en el secundario con respecto al voltaje en el primario $\left(\mathrm{V}_{\text {out }} / \mathrm{V}_{\text {in }}\right)$ de los 3 transformadores en vacío, lo cual se realizó a través del programa computacional desarrollado en Matlab (Velilla, 2011), cambiando la frecuencia de la onda sinusoidal del generador de señales AFG3021B entre $10 \mathrm{~Hz}$ a $1 \mathrm{MHz}$ (Fig. 2a). Estas señales sinusoidales son aplicadas en el primario de los transformadores $\left(V_{i n}\right)$ y observadas en el secundario como señales de salida $\left(\mathrm{V}_{\text {out }}\right)$, ambas señales son adquiridas por el programa computacional a través del osciloscopio digital TDS2022B de Tektronix. Las señales sinusoidales de entrada y salida en la frecuencia, son procesadas en el programa para obtener la magnitud de la relación de voltaje de salida con respecto al voltaje de entrada (ganancia de tensión $=V_{\text {out }} / V_{\text {in }}$ ), y el desfase entre la salida y la entrada, ambas en el rango de frecuencias determinado. En la Fig. 6 se presenta la RF de la ganancia de tensión experimental obtenida para los 3 transformadores.

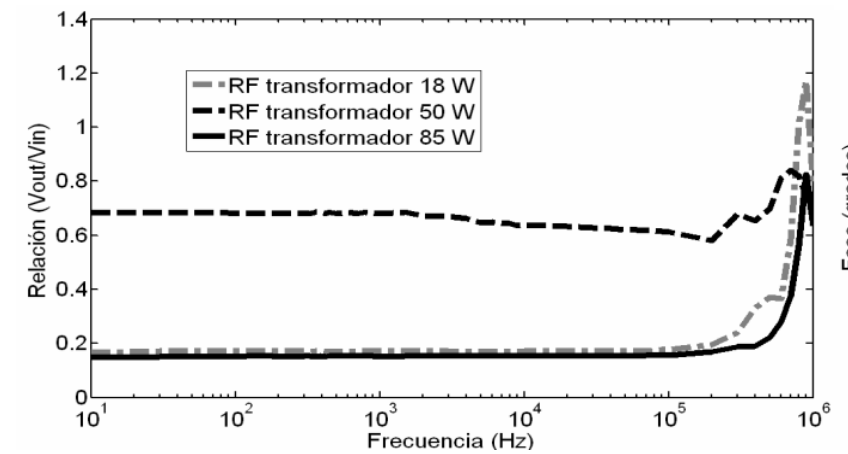

a) Ganancia de tensión

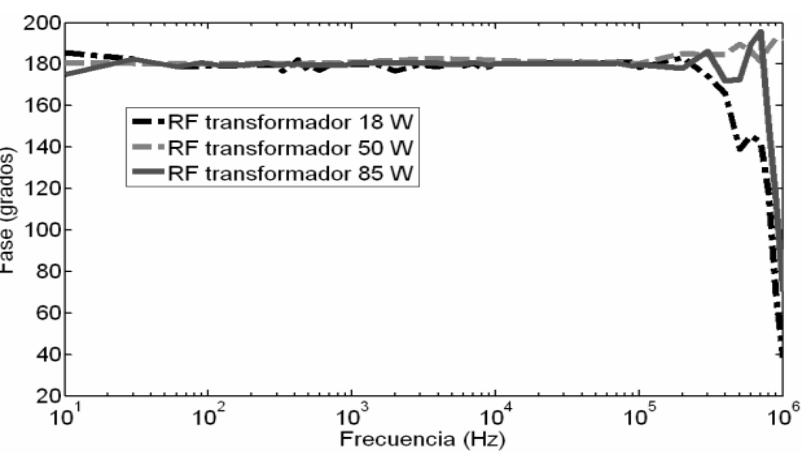

b) Fase

Fig. 6: Respuesta en frecuencia de la ganancia de tensión de los 3 transformadores

La Fig. 6 muestra algunas particularidades de la RF de los 3 transformadores de 85, 50 y 18 W: no hay variaciones significativas de la ganancia de tensión en el rango entre 10 y $10^{5} \mathrm{~Hz}$, siendo estos valores iguales a $0.1493,0.6819$ y 0.1669 respectivamente, valores próximos a las RNT mostradas en la tabla 1; el desfase es constante en el mismo rango de frecuencias en el cual la ganancia de tensión se mantiene aproximadamente constante (desfase que depende de la ubicación de las puntas del osciloscopio); la RF de la ganancia de tensión de los transformadores presenta cambios de concavidad significativos entre los $100 \mathrm{kHz}$ y $1 \mathrm{MHz}$, lo cual ocurre tanto en la ganancia de tensión como en la fase; se observa un pico máximo en las RF aproximadamente en 900,700 y $900 \mathrm{kHz}$, siendo estos valores las frecuencias de resonancia de los 3 transformadores respectivamente, valores asociados con la rapidez de respuesta o amortiguamiento del sistema (Kuo, 1996), mientras menor sea la frecuencia de resonancia, el sistema responderá más lentamente, como ocurrió con el transformador de $50 \mathrm{~W}$ (Fig. 4), y mientras la frecuencia de resonancia sea mayor, el sistema responderá más rápidamente, como ocurrió con los otros 2 transformadores, para los cuales la frecuencia de resonancia fue aproximadamente la misma, y por tanto, el comportamiento oscilatorio de los impulsos transferidos son muy similares (Fig. 3 y 5 ). Adicionalmente, la RF brinda información sobre la robustez de los transformadores, siendo un sistema más robusto en la medida que no presente variaciones significativas en la RF, 
observándose en la Fig. 6 que la curva más plana es la del autotransformador de 50 W, y por tanto éste sería el más robusto, mientras que la RF del transformador de $18 \mathrm{~W}$, es la que presenta más variaciones, y por tanto, sería el menos robusto.

\section{Ajuste de la ganancia de tensión a funciones de transferencia}

La RF puede ser utilizada para realizar diagnóstico en transformadores, técnica FRA o SFRA (Secue, 2008), o para obtener modelos matemáticos bien sean circuitos eléctricos o funciones de transferencia (Velilla, 2008). En este trabajo se optó por ajustar las RF mostradas en la Fig. 6 de los 3 transformadores en vacío, a funciones de transferencia (FT) utilizando Vector Fitting (VF) (Gustavsen, 2006) e InvFreqs (InvF) (Matlab, 2011), estableciendo como FT la relación entre el voltaje del secundario $\mathrm{V}(\mathrm{s})_{\text {out }}$ con respecto al voltaje aplicado en el primario del transformador $\mathrm{V}(\mathrm{s})_{\text {in, }}$, en términos del operador de Laplace (s), esto es, una función racional de los polinomios numerador y denominador en términos de s, ecuación 1.

$\mathbf{F T}(s)=\frac{V(s)_{\text {out }}}{V(s)_{\text {in }}}=\frac{\mathrm{a}_{\mathbf{n}} \mathrm{s}^{\mathrm{n}}+\mathrm{a}_{\mathrm{n}-1} \mathrm{~s}^{\mathrm{n}-1}+\cdots \mathrm{a}_{1} s+\mathrm{a}_{0}}{\mathrm{~b}_{\mathrm{n}} \mathrm{s}^{\mathrm{n}}+\mathrm{b}_{\mathrm{n}-1} \mathrm{~s}^{\mathrm{n}-1}+\cdots \mathrm{b}_{1} s+\mathrm{b}_{0}}$

El ajuste de la RF a FT consistió en encontrar los coeficientes que acompañan las respectivas potencias de $\mathrm{s}$, tanto del numerador como del denominador de la ecuación 1 , lo cual se realizó considerando FT propias (orden del numerador igual al del denominador) de orden 6 y 9 , debido a que en trabajos previos, se había realizado un análisis de sensibilidad en cuanto al orden de las FT que mejor representaban la RF de la ganancia de tensión de transformadores monofásicos (Velilla, 2011). La métrica de evaluación del desempeño de las FT en el dominio de la frecuencia fue el error medio cuadrático (Gustavsen, 2006), y los errores obtenidos se presentan en la tabla 2; observándose que los errores obtenidos con InvF, son los más bajos.

Tabla 2: Errores medios cuadráticos en los ajustes

\begin{tabular}{|c|c|c|c|c|c|c|}
\hline \multirow{2}{*}{ Orden de la FT } & \multicolumn{2}{|c|}{$85 \mathrm{~W}$} & \multicolumn{2}{c|}{$50 \mathrm{~W}$} & \multicolumn{2}{c|}{$18 \mathrm{~W}$} \\
\cline { 2 - 7 } & InvF & VF & InvF & VF & InvF & VF \\
\hline 6 & 0.00092 & 0.01995 & 0.00432 & 0.03775 & 0.00295 & 0.03157 \\
\hline 9 & 0.00130 & 0.01937 & 0.00134 & 0.03894 & 0.00105 & 0.01090 \\
\hline
\end{tabular}

En la Fig. 7 se presentan los ajustes de la RF experimental del transformador de $85 \mathrm{~W}$ a las FT utilizando VF e InvF, mostrándose que ambos ordenes con VF dan resultados muy similares, mientras que la FT de orden 6 de InvF, sigue mejor la tendencia de la RF experimental. En la Fig. 8 se observan los resultados obtenidos al ajustar las FT con la RF experimental del transformador de $50 \mathrm{~W}$, mostrándose que éstas no permiten reproducir adecuadamente la RF experimental, sin embargo, los resultados de InvF se aproximan mejor a los experimentales. Por último, en la Fig. 9 se muestran los resultados obtenidos con las FT ajustadas con la RF del transformador de $18 \mathrm{~W}$, resultados que indican que ambas técnicas reproducen adecuadamente la RF experimental, teniendo un mejor desempeño las FT de orden 6 tanto con VF como con InvF.

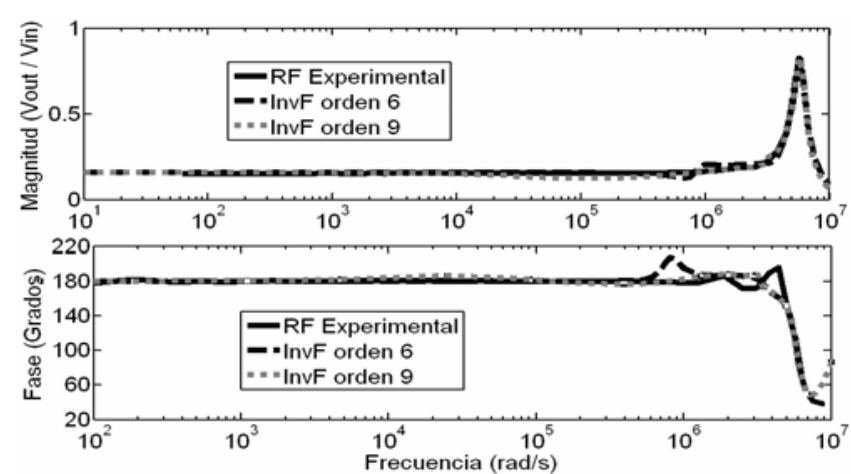

a) InvFreqs

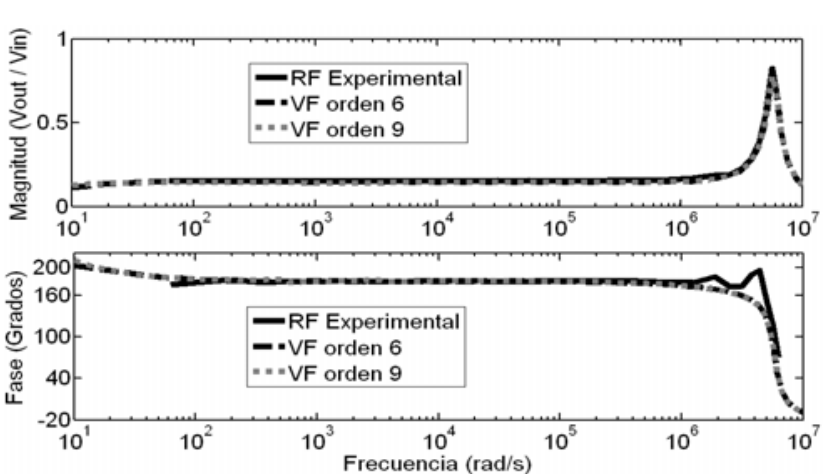

b) Vector Fitting

Fig. 7: Ajuste de las FT del transformador de $85 \mathrm{~W}$ 


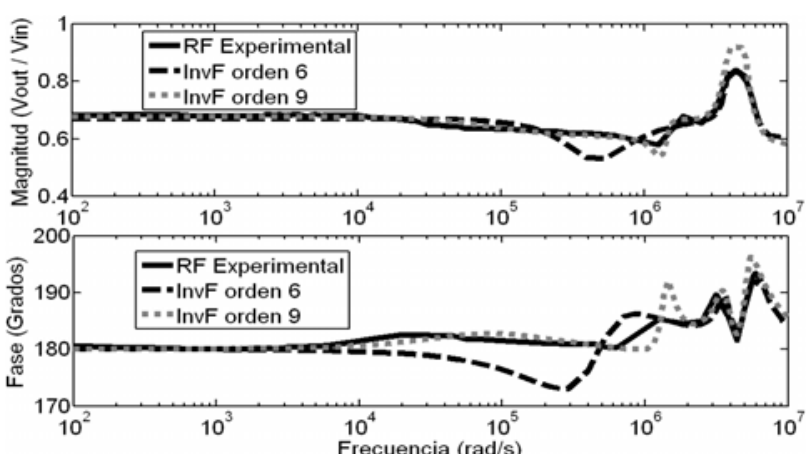

a) InvFreqs

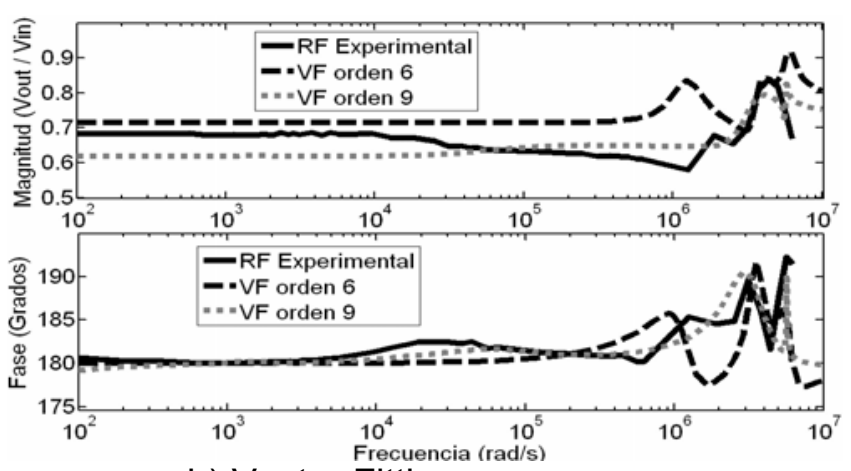

b) Vector Fitting

Fig. 8: Ajuste de las FT del autotransformador de $50 \mathrm{~W}$

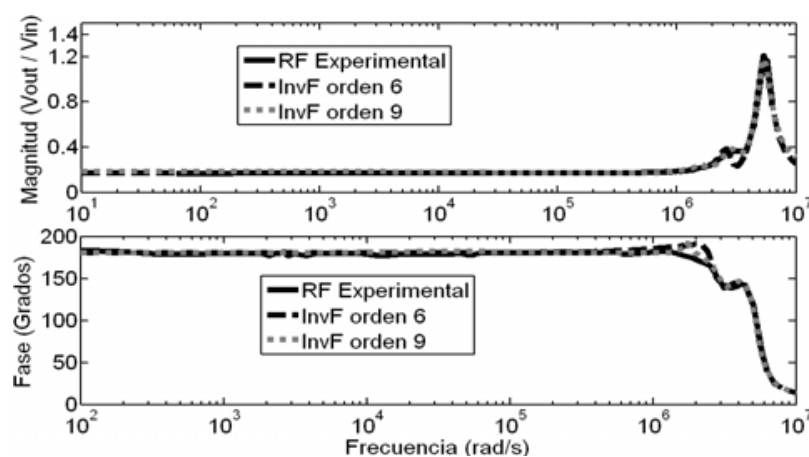

a) InvFreqs

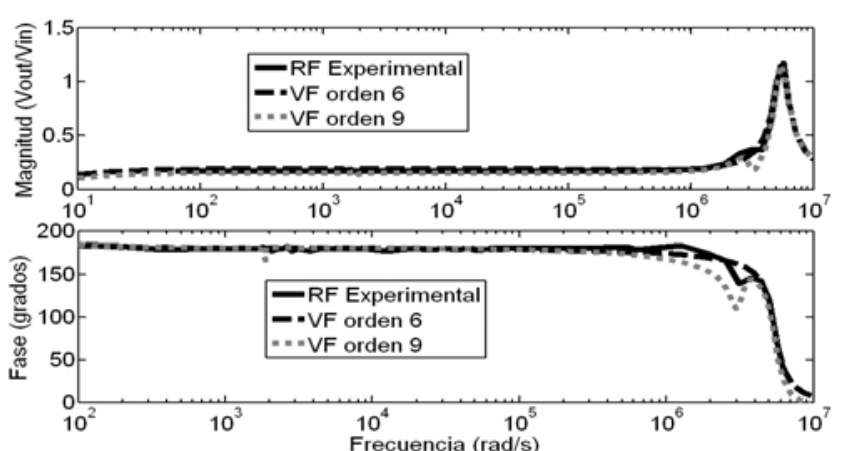

b) Vector Fitting

Fig. 9: Ajuste de las FT del transformador de $18 \mathrm{~W}$

Los resultados de las Fig. 7, 8 y 9, muestran que las FT de orden 6 describen mejor la tendencia de las RF experimentales en todo el rango de frecuencia de interés, por esta razón en la Tabla 3 solo se presentan los coeficientes de estas FT, siendo $a_{i}$ los coeficientes del numerador y $b_{i}$ los coeficientes del denominador, encontrándose coeficientes relativamente grandes (hasta del orden de $2.34 \mathrm{e}^{39}$ ), afectando estas magnitudes principalmente la ubicación de las raíces del numerador (ceros) y del denominador (polos) en el plano complejo, estas raíces serán las encargadas de la dinámica del sistema (Kuo, 1996).

Tabla 3: Coeficientes de las FT de orden 6

\begin{tabular}{|c|c|c|c|c|c|c|c|c|}
\hline \multicolumn{2}{|c|}{ Transformador } & $s^{6}$ & $S^{5}$ & $S^{4}$ & $S^{3}$ & $S^{2}$ & $\mathrm{~S}^{1}$ & $S^{0}$ \\
\hline \multirow{4}{*}{$85 \mathrm{~W}$} & $V F-a_{i}$ & -0.1727 & $-6.0811 e^{4}$ & $-9.5912 e^{8}$ & $-6.09 e^{11}$ & $-2.70 e^{14}$ & $-7.83 e^{16}$ & $-6.94 e^{17}$ \\
\hline & $V F-b_{i}$ & 1.0000 & $3.1458 \mathrm{e}^{5}$ & $4.9412 \mathrm{e}^{9}$ & $3.10 \mathrm{e}^{12}$ & $1.37 \mathrm{e}^{15}$ & $3.94 \mathrm{e}^{17}$ & $1.53 e^{18}$ \\
\hline & InvF- $a_{i}$ & 0.2509 & $10.837 e^{5}$ & $5.5125 e^{12}$ & $5.88 e^{19}$ & $4.01 \mathrm{e}^{25}$ & $3.84 \mathrm{e}^{32}$ & $3.41 \mathrm{e}^{36}$ \\
\hline & InvF- $b_{i}$ & 1.0000 & $95.879 e^{5}$ & $1.9293 e^{13}$ & $4.14 \mathrm{e}^{20}$ & $1.63 e^{26}$ & $2.57 \mathrm{e}^{33}$ & $2.11 \mathrm{e}^{37}$ \\
\hline \multirow{4}{*}{$50 \mathrm{~W}$} & $V F-a_{i}$ & -0.7851 & $-27.038 e^{5}$ & $-4.033 e^{13}$ & $-7.75 e^{19}$ & $-4.20 e^{26}$ & $-3.45 e^{32}$ & $-4.78 e^{38}$ \\
\hline & $V F-b_{i}$ & 1.0000 & $31.543 e^{5}$ & $5.2256 \mathrm{e}^{13}$ & $9.34 \mathrm{e}^{19}$ & $5.66 \mathrm{e}^{26}$ & $4.16 \mathrm{e}^{32}$ & $6.69 \mathrm{e}^{38}$ \\
\hline & InvF- $a_{i}$ & 0.6143 & $-7.6720 e^{5}$ & $2.6625 \mathrm{e}^{13}$ & $9.92 \mathrm{e}^{18}$ & $2.87 \mathrm{e}^{26}$ & $1.42 \mathrm{e}^{32}$ & $6.10 \mathrm{e}^{37}$ \\
\hline & InvF- $b_{i}$ & 1.0000 & $-7.6599 e^{5}$ & $4.1388 \mathrm{e}^{13}$ & $2.00 \mathrm{e}^{19}$ & $4.35 \mathrm{e}^{26}$ & $2.70 \mathrm{e}^{32}$ & $9.12 \mathrm{e}^{37}$ \\
\hline \multirow{4}{*}{$18 \mathrm{~W}$} & $V F-a_{i}$ & -0.1430 & $-2.2742 e^{5}$ & $5.3259 e^{12}$ & $1.16 \mathrm{e}^{18}$ & $1.85 \mathrm{e}^{20}$ & $3.65 \mathrm{e}^{24}$ & $2.51 \mathrm{e}^{25}$ \\
\hline & $V F-b_{i}$ & 1.0000 & $17.487 e^{5}$ & $3.0438 e^{13}$ & $6.22 \mathrm{e}^{18}$ & $1.05 \mathrm{e}^{21}$ & $1.96 \mathrm{e}^{25}$ & $2.54 \mathrm{e}^{26}$ \\
\hline & InvF- $a_{i}$ & 0.1310 & $-2.5836 e^{5}$ & $-2.470 e^{12}$ & $-4.83 e^{18}$ & $-7.35 e^{25}$ & $-4.17 e^{31}$ & $-3.99 e^{38}$ \\
\hline & InvF- $b_{i}$ & 1.0000 & $5.5752 e^{5}$ & $4.6277 e^{13}$ & $-1.46 e^{18}$ & $5.98 e^{26}$ & $3.05 \mathrm{e}^{31}$ & $2.34 \mathrm{e}^{39}$ \\
\hline
\end{tabular}




\section{Validación de las FT en el dominio del tiempo}

En la sección anterior se mostró que se pueden encontrar varias FT que tratan de reproducir la RF experimental, destacándose algunas por presentar los errores más bajos o reproducir adecuadamente la tendencia de las RF experimentales, sin embargo el objetivo principal de realizar el ajuste de la RF a FT, es el de establecer modelos matemáticos que permitan observar la transferencia de tensión en los 3 transformadores para diferentes ondas de excitación. En las Fig. 10, 11 y 12 se presentan los resultados de evaluar las FT obtenidas en la sección anterior, con las ondas tipo impulso $1.2 / 50 \mu \mathrm{s}$ y la $3 / 23 \mu \mathrm{s}$, ambas ondas con una amplitud de $2 \mathrm{~V}$ pico-pico (Fig. 2b), estos resultados muestran que las FT ajustadas con VF, magnifican el impulso transferido, mientras que las FT obtenidas con InvF, reproducen mejor la transferencia del impulso de tensión medido experimentalmente en el intervalo de tiempo evaluado.

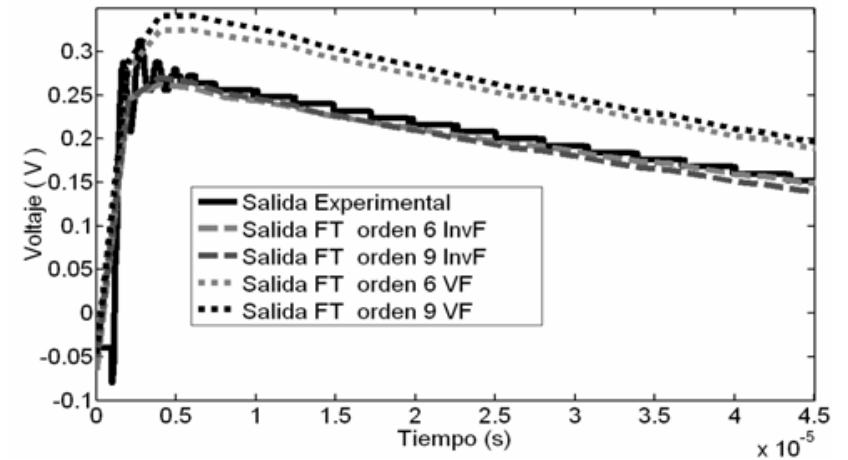

a) Impulso $1.2 / 50 \mu \mathrm{s}$

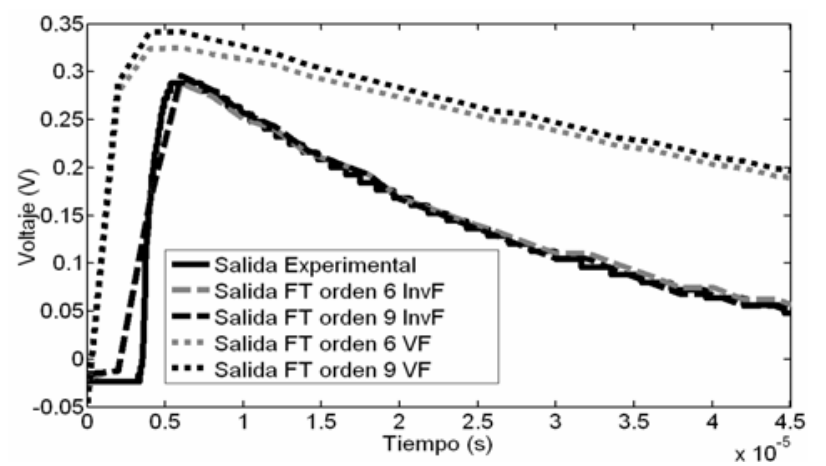

b) Impulso 3/23 $\mu \mathrm{s}$

Fig. 10: Respuesta de las FT del transformador de $85 \mathrm{~W}$ ante los impulsos

En la Fig. 11 no se incluyen los resultados de la FT de orden 9 de InvF, debido a que su comportamiento era inestable, similarmente ocurrió con la FT de orden 9 de InvF del transformador de $18 \mathrm{~W}$ (Fig. 12b), la inestabilidad de esta FT se evidencia en la Fig. 12a, debido a que la respuesta obtenida se empieza aleja considerablemente de la experimental. El problema de la inestabilidad de la técnica de InvF, es debido a que ésta no garantiza la ubicación de los polos en el semiplano izquierdo (Kuo, 1996), mientras, que con VF, si se puede restringir la ubicación de los polos al semiplano izquierdo y así garantizar el cumplimiento del criterio de estabilidad (Gustavsen, 2006).

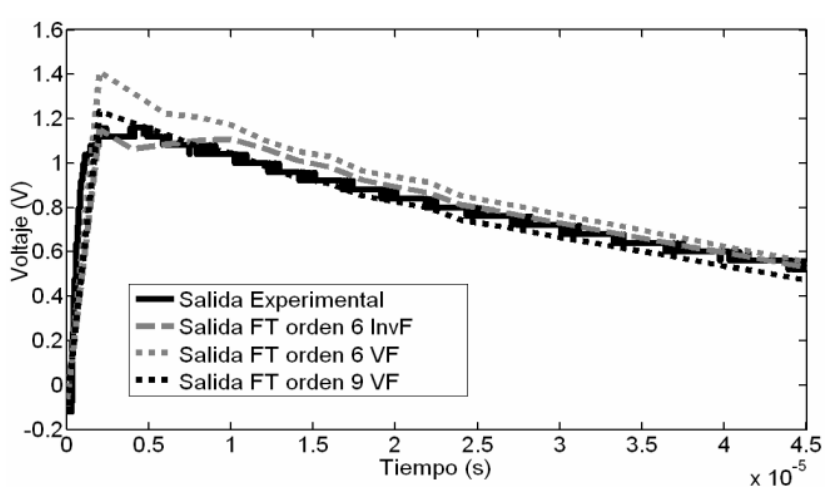

a) Impulso $1.2 / 50 \mu \mathrm{s}$

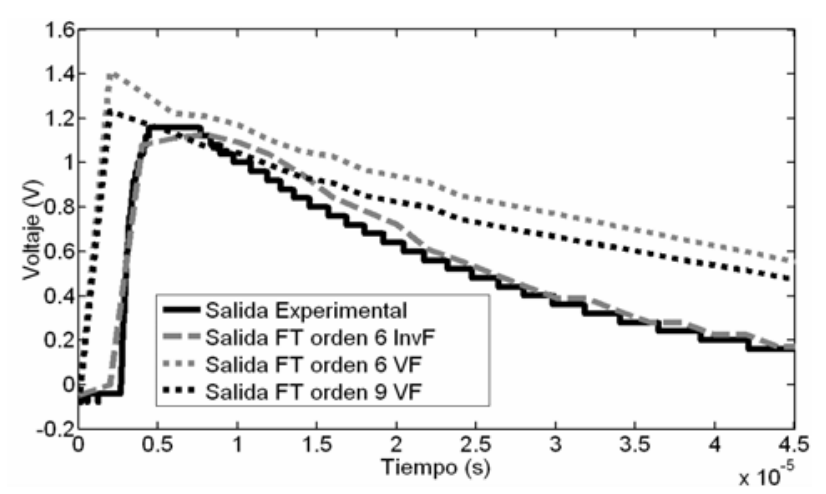

b) Impulso $3 / 23 \mu \mathrm{s}$

Fig. 11: Respuesta de las FT del transformador de $50 \mathrm{~W}$ ante los impulsos

\section{DISCUSIÓN}

Se evaluó la transferencia de impulsos de tensión experimentalmente en 3 transformadores monofásicos de baja potencia usados en aplicaciones electrónicas: uno de $85 \mathrm{~W}$; otro de $50 \mathrm{~W}$ (autotransformador) y otro de $18 \mathrm{~W}$, considerándolos en vacío y en 3 condiciones de carga 
resistiva en el secundario (16, 10 y $4 \Omega$ ), mostrándose que cuando los impulsos de tensión 1.2/50 y $3 / 23$ us fueron aplicados en el primario, la mayor transferencia de tensión en el secundario ocurrió para la condición de vacío, y en la medida que el valor de la resistencia del secundario disminuía, la transferencia de tensión en el secundario también lo hacía.

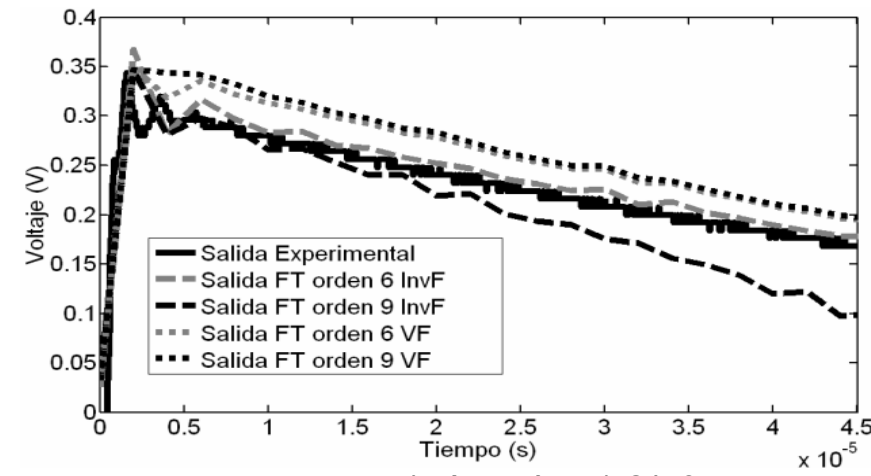

a) Impulso $1.2 / 50 \mu \mathrm{s}$

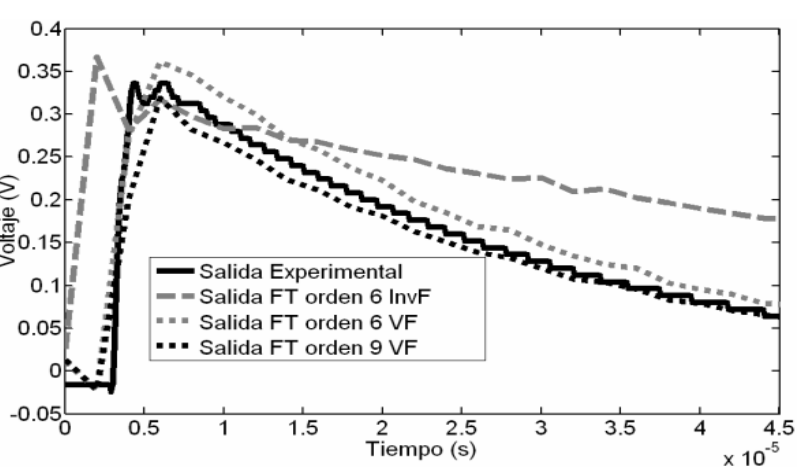

b) Impulso $3 / 23 \mu \mathrm{s}$

Fig. 12: Respuesta de las FT del transformador de $18 \mathrm{~W}$ ante los impulsos

Se observó que los impulsos transferidos en el autotransformador de $50 \mathrm{~W}$, eran los más lentos y amortiguados para ambas señales de prueba $(1.2 / 50$ y $3 / 23 \mu \mathrm{s})$, mientras que el comportamiento de los impulsos transferidos en los otros 2 transformadores fueron más rápidos y similares entre sí; lo cual es explicado a través de la respuesta en frecuencia de la ganancia de tensión $\left(V_{\text {out }} / V_{\text {in }}\right)$ de los 3 transformadores (Fig. 6), debido a que la rapidez de respuesta de los transformadores está relacionada con la frecuencia de resonancia de la ganancia de tensión, correspondiendo la respuesta más lenta al sistema con menor frecuencia de resonancia, siendo ésta la del autotransformador de $50 \mathrm{~W}(700 \mathrm{kHz})$, mientras que la frecuencia de resonancia de los otros 2 transformadores fue aproximadamente igual para ambos $(900 \mathrm{kHz})$, y por tanto, al ser ésta mayor a la del autotransformador, los impulsos transferidos fueron más rápidos.

Se obtuvo las RF de los 3 transformadores (85, 50 y $18 \mathrm{~W}$ ) entre $10 \mathrm{~Hz}$ y $1 \mathrm{MHz}$, observándose un comportamiento constante de la ganancia de tensión $\left(V_{\text {out }} / V_{\text {in }}\right)$ entre 0 y $500 \mathrm{kHz}$, valores que se aproximan a la RNT de cada transformador (Tabla 1). Este comportamiento se ve reflejado en el máximo pico de tensión transferido, encontrándose que en la medida que la RNT aumentaba, la relación pico máximo con respecto a la RNT disminuía, razón por la cual el autotransformador de $50 \mathrm{~W}$ que posee la máxima RNT de los 3 transformadores (RNT $=0.54$ ), presentó la menor relación (2.29), valor que coincidió para las 2 ondas tipo impulso de prueba evaluadas, mientras que la mayor relación fue de 3.48 y ocurrió para el transformador de $85 \mathrm{~W}$, el cual posee una RNT de 0.14. En el caso de los transformadores de 85 y $18 \mathrm{~W}$, los picos máximos de los impulsos transferidos dependieron de la excitación, siendo mayores los picos de tensión asociados a la onda 1.2/50 $\mu \mathrm{s}$, debido a que su tiempo de frente es más rápido.

Se ajustó la RF medida experimentalmente de los 3 transformadores a FT propias de orden 6 y 9 con VF e InvF, observándose que las FT de orden 6 de InvF a pesar de poseer los errores más altos en los ajustes realizados en el dominio de la frecuencia (Tabla 2), fueron las que mejor reprodujeron la transferencia de los impulsos de tensión en el dominio del tiempo para ambas señales de prueba $(1.2 / 50 \mu \mathrm{s}$ y $3 / 23 \mu \mathrm{s})$. Sin embargo, la mayoría de FT obtenidas con InvF de orden 9, presentaron problemas de estabilidad; debido a que la técnica no permite garantizar la ubicación de los polos en el semiplano izquierdo, y por tanto, no permite garantizar la estabilidad del sistema, a diferencia de la técnica de VF, la cual si permite hacerlo.

\section{CONCLUSIONES}

Con base en los resultados y discusión se pueden plantear las siguientes conclusiones principales: i) La mayoría de las funciones de transferencia obtenidas con InvF de orden 9, 
presentaron problemas de estabilidad y por tanto, no permite garantizar la estabilidad del sistema, a diferencia de la técnica de ajuste vectorial (vector fitting, VF), la cual si permite hacerlo; ii) La máxima transferencia de tensión se presentó cuando los transformadores están en vacío; y iii) La comparación de los resultados obtenidos en el dominio del tiempo con los impulsos de tensión aplicados, evidenció la importancia de la respuesta en frecuencia; de la carga en el secundario y de la forma de onda del impulso aplicado, en el impulso transferido al secundario.

\section{REFERENCIAS}

De Conti, A. y S. Visacro, "Evaluation of lightning surges transferred from medium voltage to low voltage networks". Generation, Transmission and Distribution, IEEE Proceedings. 152(3), 351 356 (2005).

Das, J.C., Surge transference through transformers, Industry Applications Magazine, IEEE, 9(5), 24-32 (2003).

Gustavsen, B., Improving the Pole Recolating properties of Vector Fitting, IEEE Transaction on Power Delivery, 21(3), 1587 - 1592 (2006).

Kanashiro, A., A. Piantini y G.F. Burani. Modelo de Transformador de Distribución Trifásico para Estudios de Máximos de Tensión (Peaks) Ocasionados por Descargas Atmosféricas. Información Tecnológica, 15(1), 67-73 (2004).

Kuo, B., Sistemas de Control Automáticos, Séptima Edición, 509-645. Pearson, Prentice Hall. México (1996).

Matlab Web Site, Toolbox of Signal Processing.

http://www.mathworks.com/help/toolbox/signal/InvFreqs.html. Acceso: 10 de Marzo (2011).

Noda, M., M. Sakae y S. Yokoyama, Simulation of lightning surge propagation from distribution line to consumer entrance via pole-mounted transformer, IEEE Trans. On Power Delivery, 19(1), 442444 (2004).

NTC 4552-1, Norma Técnica Colombiana, Protección contra Descargas Eléctricas Atmosféricas (Rayos), Parte 1: Principios Generales, 29-37, Bogotá, Colombia (2008).

Obase, P.F., Surtos Atmosféricos transferidos à rede secundária via transformador, Mestre em engenharia, Escola Politécnica da Universidade de São Paulo, São Paulo, Brasil (2004).

Okabe, S., Koto M., Ueta G., Development of High Frequency Circuit Model for Oil-immersed Power Transformers and its Application for Lightning Surge Analysis, IEEE Transactions on Dielectrics and Electrical Insulation, 18(2), 541-552 (2011).

Secue, J. y E. Mombelo, Sweep frequency response analysis (SFRA) for the assessment of winding displacements and deformation in power transformers, Electric Power Systems Research: 78(6), 1119-1128 (2008).

Velilla, E. y otros 4 autores, Metodología para obtener el circuito equivalente del transformador monofásico de distribución para la evaluación de impulsos transferidos por rayos a la red secundaria, Vicerrectoría de Investigación, Universidad de Antioquia, Medellín, Colombia (2011).

Velilla, E., Valencia J., Moreno G., Using Genetic Algorithm and the Simplex Method to Obtain Equivalent Circuits of the Grounding Systems, Transmission and Distributions Conference and Exposition, IEEE/PES, Bogotá, Colombia, 13 al 15 de Agosto (2008). 